\title{
Investigation on fracture of pre-cracked single-layer graphene sheets
}

\author{
Xuefeng Liu ${ }^{\mathrm{a}}$, Zhiwu Bie ${ }^{\mathrm{b}}$, Jinbao Wang ${ }^{\mathrm{c}}$, Ligang Sun ${ }^{\mathrm{d}}$, Meiling Tian ${ }^{\mathrm{c}}$, Erkan Oterkus ${ }^{\mathrm{e}}$, Xiaoqiao He ${ }^{\mathrm{a}, \mathrm{b}^{*}}$ \\ ${ }^{a}$ Department of Architecture and Civil Engineering, City University of Hong Kong, Hong Kong SAR, China \\ ${ }^{\mathrm{b}}$ City University of Hong Kong (Shenzhen) Research Institute, Shenzhen, China \\ ${ }^{\mathrm{c}}$ School of Port \& Transportation Engineering, Zhejiang Ocean University, Zhoushan, China \\ ${ }^{\mathrm{d}}$ Department of Mechanical and Biomedical Engineering, City University of Hong Kong, Hong Kong SAR, \\ China \\ ${ }^{\mathrm{e}}$ Department of Naval Architecture, Ocean and Marine Engineering, University of Strathclyde, Glasgow, UK
}

\begin{abstract}
Peridynamics (PD) is employed to model the fracture of pre-cracked graphene sheets under mode-I loading condition to show its application at nanoscale. Then the related mechanisms at atomic scale are revealed by using molecular dynamics (MD) simulation, with which the mechanical properties and fracture mechanisms of full atomistic pre-cracked single-layer graphene sheets (SLGSs) with different types of crack tip micro-structures are investigated. The results such as the fracture forms obtained from the PD and MD simulations show good consistency. The MD results show that different crack tip structures have distinct effect on the mechanical properties of graphene sheets. The pre-cracked SLGSs with $\omega$-type crack tip show higher fracture strength and strain than the ones with u-type crack tip. For the pre-cracked SLGSs with u-type crack tip, the fracture strength and strain of the armchair sheet are higher than the zigzag one. However, there are almost no differences for the armchair and zigzag sheets with $\omega$-type crack tip. Due to the difference between armchair and zigzag structures, carbon polygon ring can form in armchair sheets and its formation is related to the crack tip structures and the crack width. In addition, the crack propagation can be characterized by the local stress state, which for crack initiation is different from steady crack propagation.
\end{abstract}

Keywords: Single-layer graphene sheet; Fracture mechanism; Crack tip; Molecular dynamics; Peridynamics

\section{Introduction}

Graphene [1-3], a kind of unique and one-atom thick two-dimensional nano-structure, is a single

*Corresponding author: jinbaow @zjou.edu.cn(Jinbao Wang); bcxqhe@ cityu.edu.hk(Xiaoqiao He) 
layer of carbon atoms arranged in a honeycomb crystal lattice regularly, which is the basic element to build other graphitic materials, such as one-dimensional carbon nano-tube or three-dimensional graphite. Due to its preeminent mechanical properties [4-7], excellent electrical properties [8-11] and outstanding thermal properties [12-14], graphene has been considered as a kind of promising material, and can be widely applied in nano-electronic systems like capacitors [15-17], transparent electrodes [18-20], transistors [21, 22], photodetectors [23], conductors[24] and semiconductors [25], nano-electro-mechanical systems like sensitive sensors [26, 27], transparent interconnects [28] and electrostatic actuators [29], and nano-composite systems [30-35]. Thus, full understanding of various behaviors of graphene sheets can be very significant in designing graphene-based nano-structures.

Kam et al. [36] found the mechanical response of graphene nanofilm mainly depends on its geometry, temperature and loading conditions. Reddy et al. [37] showed that edge effect strongly influences the elastic properties of graphene with smaller width and becomes negligible for wider ones. The edge effect on fracture strength of defect-free graphene was also found by Kiselev and Wang et al. [38, 39]. Besides, they also found temperature rise and defects such as Stone-Wales defects and vacancies can cause strength loss and the fracture site of defective graphene initiating from defects. Sun et al. [40] found the fracture strength of graphene losses about $17.7 \%$ due to the monatomic vacancy. Zhu et al. [41] found that the Young's modulus of graphene is largely correlated to the size of vacancy defects perpendicular to the stretching direction and reduces at high concentration of mono-atomic-vacancy defects, and the blunting effect of vacancy edges plays an important role in the vacancy-induced crack initiation and propagation. Zhao et al. [42] found that the Young's modulus of monolayer graphene does not vary significantly with temperature until about $1200 \mathrm{~K}$, beyond which the monolayer graphene becomes softer. The results of Zhang et al. [43] showed that the mechanical properties of graphene sheets degrade linearly when temperature becomes high, and layer number and isotope-substitution lead to marginal difference in mechanical properties.

Beside of the effect of Stone-Wales or vacancy defects on the properties of graphene sheets, there are also many investigations about the mechanical properties of graphene sheets with a crack via numerical simulation methods. Tsai et al. [44] found that the strain energy release rate can be employed in atomic and continuum models to describe the fracture of covalently bonded graphene sheets, while the stress intensity factor may not be suitable for modeling the crack behavior in atomic structures. Compared to the graphene with vertical pre-crack, the ones with inclined pre-crack possess higher fracture strength and strain and smaller critical energy release rate which is independent of initial crack length at low temperature. Meanwhile, armchair 
graphene always has greater critical energy release rate than zigzag graphene, and the critical energy release rate of graphene decreases with increment of temperature [45]. Besides, the increase of temperature and crack length can lead to the reduction of fracture strength [42, 45-49]. Based on experiment and modeling, Zhang et al. [50] found that the strength of large-area graphene should be determined by its fracture toughness, rather than intrinsic strength. Le et al. [47-49] found that either the fracture strain or the crack propagation speed of the graphene with shorter initial crack is larger than that of the ones with longer initial crack in pre-cracked graphene. While in pre-strained graphene sheets, crack propagation speed is independent of the length, location and number of inserted crack. For the graphene sheet with a hole in its center, Fang et al. [51, 52] found that the fracture strength, strain and Young's modulus decrease while the Poisson's ratio increases with the hole size increasing. For the graphene sheet with v-shape crack, they also found that the crack propagates from the crack tip along the direction perpendicular to the loading axis for armchair sheet and along the direction of $45^{\circ}$ from the loading axis for zigzag sheet. The zigzag one can bear larger load and its fracture energy is also larger and decreases with the increase of crack length. However, different from the results of Fang et al., $\mathrm{Xu}$ et al. [53] found the zigzag crack evolves along a $0^{\circ}$ angle in a straight line and the armchair crack propagates by oscillating between $\pm 30^{\circ}$ angles of the initial crack direction under mode I loading. In addition, the vacancy defects existing in graphene sheets can affect the crack propagation trajectory as well [41, 54]. Interestingly, Parashar et al. [55] showed that van der Waals interaction forces have significant impact on the opening mode fracture characteristics of graphene sheet when inter-layer spacing is $0.344 \mathrm{~nm}$. And when inter-layer spacing is larger than $0.344 \mathrm{~nm}$, its effect starts mitigating with inter-layer spacing increasing and ultimately becomes negligible after inter-layer spacing beyond $0.3816 \mathrm{~nm}$.

In addition, as a new continuum-based theory, PD theory [56] can be a convenient method for discontinuous problems such as crack propagation for the reason that the PD equation of motion is free of the spatial derivatives of the displacement field. And recent works by Oterkus et al. [57] and Martowicz et al. [58] show that the PD theory can also be employed in the study on graphene sheet at nanoscale. Oterkus et al. [57] studied the fracture of pre-cracked graphene sheets and showed that the PD theory can be a more efficient method compared with the MD simulation. However, the fracture mechanism at atomic scale cannot be revealed by using the PD-based simulations.

In this work, the fracture behavior in pre-cracked graphene sheets is modeled by using the PD theory and the results are compared with the ones from MD simulations. To show more mechanical mechanisms at atomic scale, this study also studies the mechanical properties of full 
atomistic pre-cracked single-layer armchair and zigzag graphene sheets via MD simulations, and reveals the mechanism of crack propagation in detail. Thus, it provides a better understanding of the mechanical properties of such kind of graphene sheets, which will be helpful for utilizing them in nano-systems.

\section{Computational methodology}

\subsection{Peridynamics-based simulations}

The ordinary state-based peridynamic (OSPD) theory [59] is employed to model the fracture of pre-cracked graphene sheet. In the theory, a continuum medium is composed of infinitesimal material points and each material point interacts with other points inside its influence domain, $H$, called horizon with radius of $\delta$ as shown in Fig. 1. In the figure, it shows the configurations before and after deformation, in which $\mathbf{x}_{k}, \mathbf{x}_{j}$ and $\mathbf{y}_{k}, \mathbf{y}_{j}$ stand for the position vectors of material points $k$ and $j$ in the undeformed and deformed configurations, respectively. $\mathbf{t}_{k j}$ and $\mathbf{t}_{j k}$ represent the PD force density vector on material point $k$ from point $j$ and on material point $j$ from $k$, respectively.

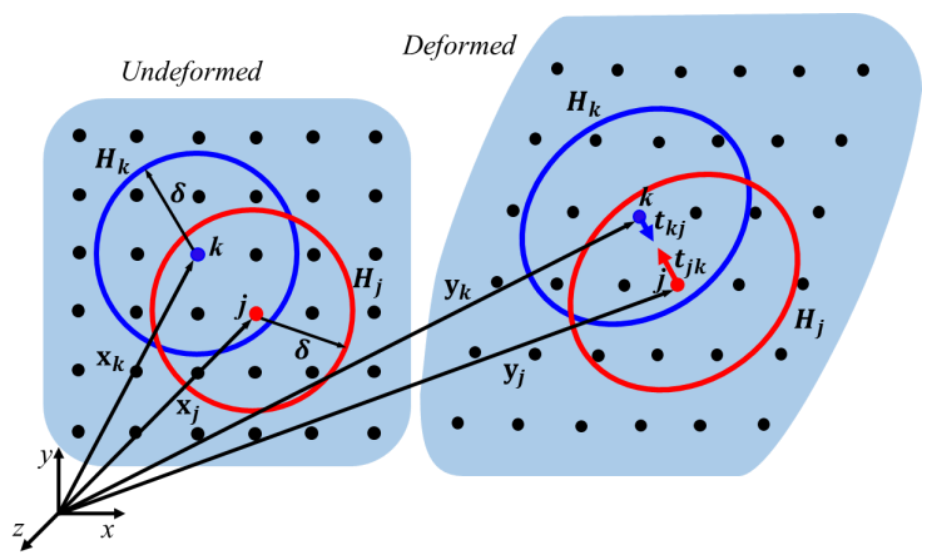

Fig. 1 Schematic shows the interaction between material points $k$ and $j$ in the horizon, $H$.

The PD equation of motion of material point $k$ is expressed as [59]

$$
\rho_{k} \ddot{\mathbf{u}}_{k}=\sum_{j=1}^{N}\left(\mathbf{t}_{k j}-\mathbf{t}_{j k}\right) V_{j}+\mathbf{b}_{k}
$$

in which $\mathbf{t}_{k j}-\mathbf{t}_{j k}$ is the net PD force density vector acting on material point $k, \rho_{k}$ is the density, $\ddot{\mathbf{u}}_{k}$ is the acceleration, $\mathbf{b}_{k}$ is the body force density, $N$ is the number of material points in the horizon of the material point $k$ and $V$ represents the volume of the material point. The PD force density vector at material point $k$ can be expressed by the corresponding strain energy 
density function, $W_{k}$, as [59]

$$
\mathbf{t}_{k j}=\frac{1}{V_{j}} \frac{\partial W_{k}}{\partial\left(\left|\mathbf{y}_{j}-\mathbf{y}_{k}\right|\right)} \frac{\mathbf{y}_{j}-\mathbf{y}_{k}}{\left|\mathbf{y}_{j}-\mathbf{y}_{k}\right|}
$$

where $|\cdot|$ denotes the length of the corresponding vector. An analogous expression also holds for $\mathbf{t}_{j k}$. Based on the PD integral form of strain invariant [60], the strain energy density of material point $k$ can be derived and expressed as [61]

$$
W_{k}=\left(a_{1} \theta_{k}+a_{0}\right) \theta_{k}^{2}+\left(b_{1} \theta_{k}+b_{0}\right) \sum_{j=1}^{N} w_{k j}\left(\left|\mathbf{y}_{j}-\mathbf{y}_{k}\right| \Lambda_{k j}-\left|\mathbf{x}_{j}-\mathbf{x}_{k}\right|\right)^{2} V_{j}
$$

in which $\theta_{k}$ is the dilatation term of material point $k$, and $w_{k j}$ is an influence function. The dilatation can be defined as

$$
\theta_{k}=\frac{3}{\pi h \delta^{4}} \sum_{j=1}^{N} w_{k j}\left(\left|\mathbf{y}_{j}-\mathbf{y}_{k}\right| \Lambda_{k j}-\left|\mathbf{x}_{j}-\mathbf{x}_{k}\right|\right)\left|\mathbf{x}_{j}-\mathbf{x}_{k}\right| V_{j}
$$

with

$$
\Lambda_{k j}=\frac{\mathbf{y}_{j}-\mathbf{y}_{k}}{\left|\mathbf{y}_{j}-\mathbf{y}_{k}\right|} \cdot \frac{\mathbf{x}_{j}-\mathbf{x}_{k}}{\left|\mathbf{x}_{j}-\mathbf{x}_{k}\right|}
$$

and

$$
w_{k j}=\frac{\delta}{\left|\mathbf{x}_{j}-\mathbf{x}_{k}\right|}
$$

In Eq. (3), $a_{0}, a_{1}, b_{0}$ and $b_{1}$ are PD parameters, which are equal to [61]

$$
\begin{gathered}
a_{0}=-3.85 \times 10^{10} \mathrm{~J} / \mathrm{m}^{3}, a_{1}=1.89 \times 10^{11} \mathrm{~J} / \mathrm{m}^{3}, \\
b_{0}=\frac{1.93 \times 10^{12}}{\pi h \delta^{4}} \mathrm{~J} / \mathrm{m}^{8}, b_{1}=\frac{-2.80 \times 10^{12}}{\pi h \delta^{4}} \mathrm{~J} / \mathrm{m}^{8} .
\end{gathered}
$$

Zhao et al. [62] found that the size effect on the Young's modulus and the Poisson's ratio is negligible when the diagonal length of a square graphene ribbon exceeds 10nm. Therefore, a square edge pre-cracked plate model with global size of $10 \mathrm{~nm} \times 10 \mathrm{~nm}$ is used to model the fracture of graphene sheet with the OSPD theory. As a continuum based theory, each material point in the OSPD model may contain several carbon atoms, which results in the loss of information at atomic scale. Then, atomistic simulations are necessary to reveal the mechanisms at atomic scale.

\subsection{Molecular dynamics simulations}

The deformation and fracture mechanisms of graphene sheets with edge crack at atomic scale are studied via a MD open source code called Large-scale Atomic/Molecular Massively Parallel Simulator (LAMMPS) [63] and the second Reactive Empirical Bond Order (REBO) Potential [64] which is a kind of Tersoff potential to describe short-range covalent bonds of carbon atoms. The 
potential energy is calculated as

$$
\Phi_{i}=\sum_{j=1}^{N} f_{c}\left(r_{i j}\right)\left[V_{R}\left(r_{i j}\right)-\bar{b}_{i j} V_{A}\left(r_{i j}\right)\right]
$$

where $r_{i j}$ is the interatomic distance between atoms $i$ and $j, V_{R}$ and $V_{A}$ are pairwise potential functions for the repulsive and attractive interactions, respectively, $\bar{b}_{i j}$ is a bond-order term which takes the local bonding environment up to third nearest neighbors into consideration through its dependence on the bond angles, and $f_{c}$ is the cutoff function.

Comparing the PD and MD methods, it can be observed that the bond stretch and the bond angle are calculated in the MD simulations and the bond stretch and the dilatation are calculated in the PD simulations. However, the REBO potential in the MD simulations is only applicable to the fully atomic systems while the continuum-based PD method can be employed for different coarse-grained systems at nanoscale. Thus the continuum-based PD method has an obvious advantage over the MD method for the large nanoscale systems. As shown in the previous study [61], larger length scale and time step make PD a more efficient method compared with MD.

The pre-cracked graphene models are shown in Fig. 2. For computational efficiency of atomistic simulations, the size of the graphene sheet is chosen as $10 \mathrm{~nm} \times 10 \mathrm{~nm}$, which is the same as the one in the PD model. The cracks with different crack tip micro-structures are considered in the MD simulations, and their size are about $4 a \times 5.5 \sqrt{3} a$ for pre-cracked armchair sheets and $2 \sqrt{3} a \times 8 a$ for pre-cracked zigzag sheets, in which $a(a \approx 1.42 \AA)$ is the lattice constant. The crack tip micro-structures of these cracks are classified into "u-type" and " $\omega$-type", respectively. The graphene models in Figs. 2a-d are composed of 3962, 3960, 3969 and 3967 atoms, respectively. Fig. 3 shows the structures of cracks with u-type and $\omega$-type crack tips. It can be known from Fig. 3 that the crack structures above the black dotted box are the same in armchair or zigzag sheet and the cracks with $\omega$-type crack tip can be obtained by removing the two atoms which are in the red dotted ellipses and belong to the u-type crack tips.

For each case, MD simulation is performed at about $0 \mathrm{~K}$ to minimize the thermal vibration of small scale systems and the temperature of each system is kept by Nose-Hoover (NVT) thermostating [65] in the whole simulation process. At the start of simulation, an energy minimization of the system is performed by using the algorithm of conjugate gradient. Then the system is relaxed by running dynamics for 30ps without applying external loads. Finally, the relaxed system is stretched uniaxially.

In both PD and MD simulations, each case is stretched uniaxially by moving the left and right boundary layers along the arrow directions as shown in Fig. 2 with a global strain rate of $2 \times 10^{7} / \mathrm{s}$. 
The ratio of the crack length to the global size is $1 / 10$ in each case. In addition, the graphene sheet is assumed as a homogeneous continuum plate model in the PD theory and is discretized as uniformly distributed material points with spacing of $0.15 \mathrm{~nm}$, which is different from the hexagonal atom distributions in graphene sheet at atomic scale. According to the atomic structure at crack tips in the fully atomistic graphene sheets in Figs. 2a and c, different material point distributions are employed in the PD models. Two lines and one single line of material points are placed in front of the crack tips in the armchair and zigzag graphene PD models, respectively, according to the symmetry in the corresponding atomistic models. In PD simulation, the horizon size is generally chosen as 3 times of the spacing between two closest material points [59] (i.e. $\delta=0.45 \mathrm{~nm}$ in current PD simulation). While the maximum interaction distance between two atoms is $\sqrt{3} a$, or $0.246 \mathrm{~nm}$, in graphene sheet at atomic scale according to the REBO potential. The position and velocity information of each particle are updated at every timestep by employing the velocity-verlet integration scheme in both simulations [66]. The timestep used in the PD simulation can be $5 \mathrm{fs}$ [61], which is 10 times larger than the one (i.e. $0.5 \mathrm{fs}$ ) used in the MD simulation in current study. Thus, the continuum based PD theory can be more efficient than the MD simulation and is especially suitable to the study of fracture of large nanoscale systems compared with the classical continuum mechanics based methods which generally fail to solve discontinuity related problems (e.g. fracture). The results obtained from PD and MD simulations in this work are visualized by OVITO [67].
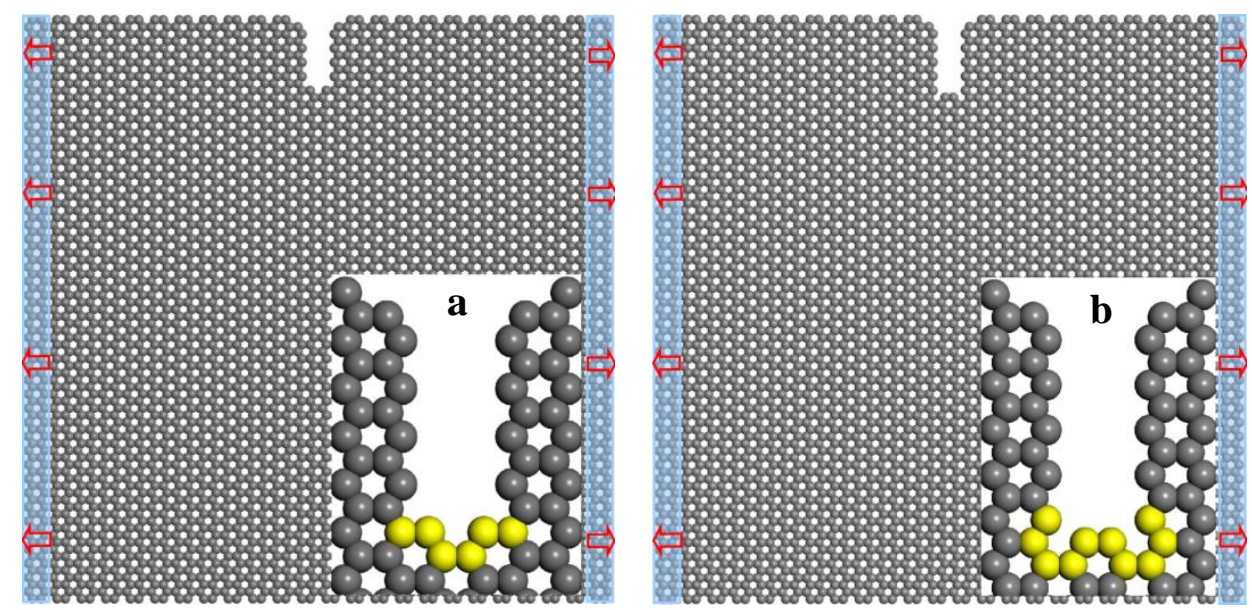

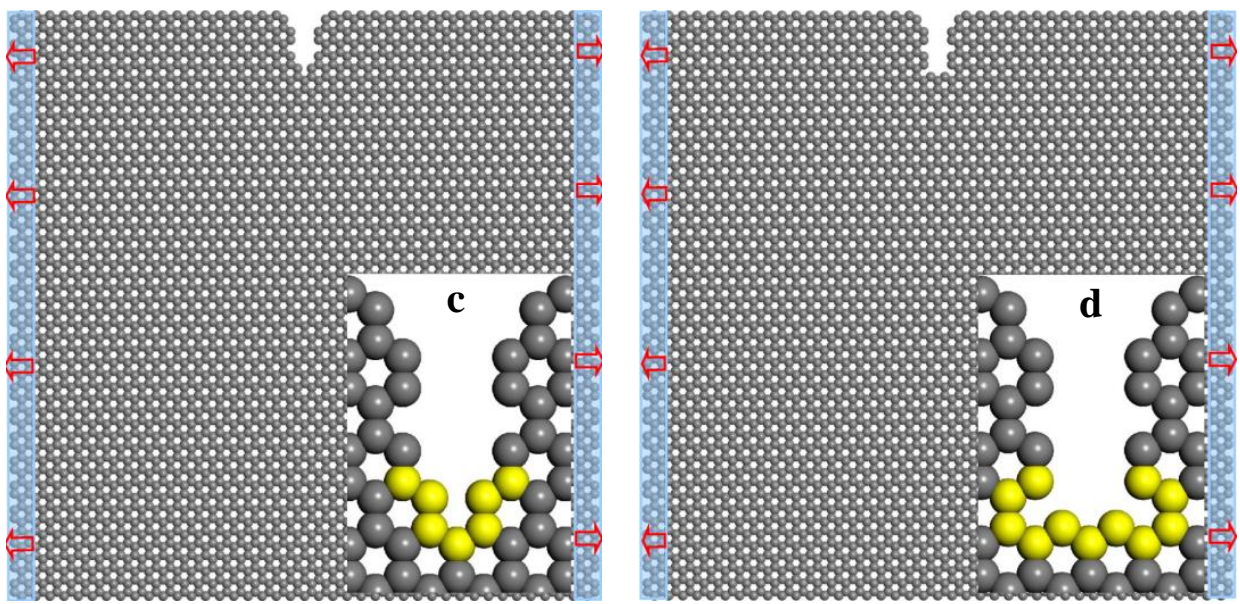

Fig. 2 The pre-cracked graphene models used in this work. (a), (b) Pre-cracked armchair models with u-type and $\omega$-type crack tips, (c), (d) Pre-cracked zigzag models with u-type and $\omega$-type crack tips. The insets show the crack tip structures.
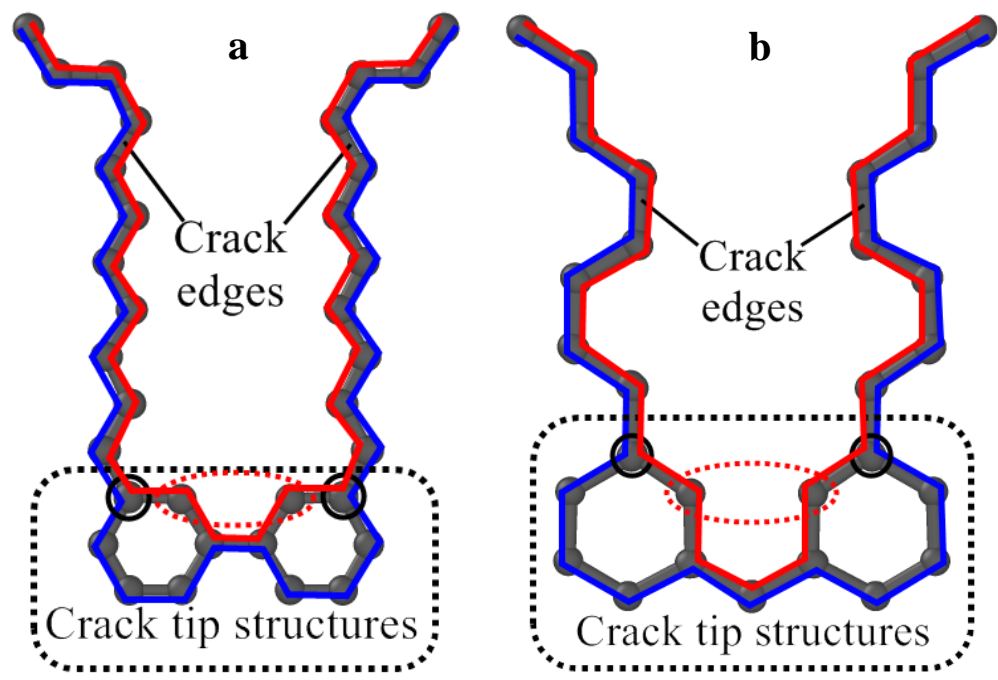

Fig.3 Structure of cracks with u-type and $\omega$-type crack tips. (a) Cracks in armchair sheets, (b) Cracks in zigzag sheets. The red lines represent the cracks with u-type crack tip and the blue lines stand for the cracks with $\omega$-type crack tip.

\section{Results and discussion}

\subsection{Comparison between PD and MD simulation results}

Fig. 4 shows the final fracture forms of pre-cracked armchair and zigzag graphene sheets. From the figure, it can be seen that brittle fracture occurs in both pre-cracked graphene sheets under mode I loading condition and that the global crack paths obtained from the PD and MD simulations show good consistency with each other for both graphene sheets, i.e. the crack in both graphene sheets propagates forward in a globally straight way and the graphene sheets break into 
two halves globally in each case. From Figs. $4 \mathrm{a}$ and c, it can be seen that the cracks are symmetric despite the effect of different material point distributions. However, the cracks are not symmetric in the fully atomistic graphene sheets because of the high frequency vibration of atoms in the thermostating process. Besides, it can be seen from the MD simulation results (Figs. 4b and d) that the local fracture forms in both graphene sheets are in a zigzag way, which is not observed from the PD simulation results for the reason that the atomic structure cannot be considered in the continuum based PD theory. In addition, the crack branching behaviors appeared in pre-cracked zigzag graphene sheets $[57,61,68]$ are not observed as well in this work, which may be due to the size effect (e.g. crack size and specimen size).

Therefore, the global fracture characteristics such as straight crack path and crack branching behaviors [61] in the propagation process of crack can be produced by using PD theory. In this respect, PD theory is applicable to the predictions of global fracture in relatively large nanoscale systems in which the fracture at atomic scale is not in consideration. Otherwise, fully atomistic simulations are needed to study the fracture at atomic scale, such as the effect of different atomic structures at crack tips and the stretching and rotation of covalent bonds in the fracture process. Thus, combination of PD and MD methods can provide a significant insight of multiscale modeling.
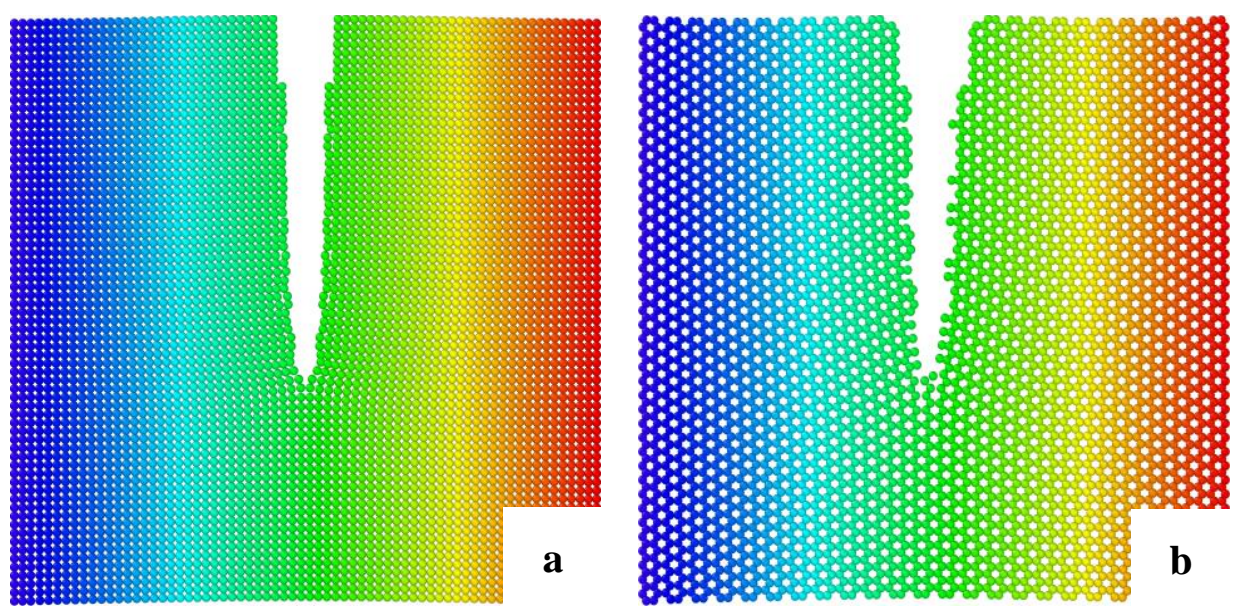

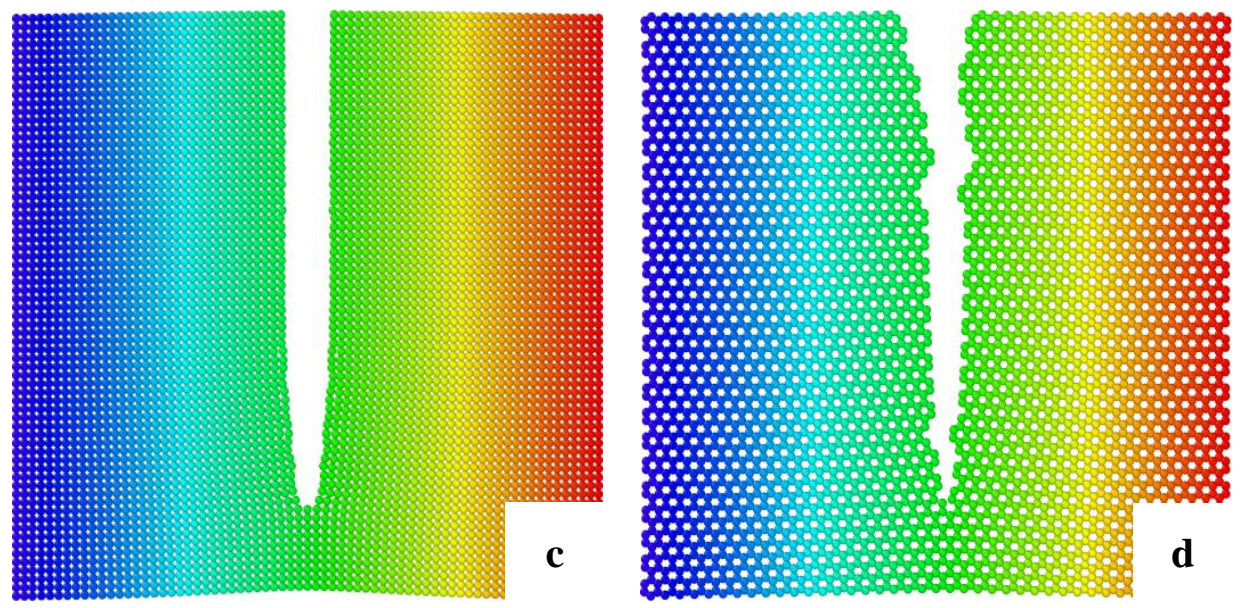

Fig. 4 The fracture patterns of pre-cracked (a), (b) armchair and (c), (d) zigzag graphene sheets obtained from (a), (c) PD and (b), (d) MD simulations. The color map represents the position distribution.

\subsection{The related mechanisms at atomic scale}

It can be known from above that the global fracture characters of pre-cracked graphene sheets can be well modeled by using PD simulation. However, the fracture at atomic scale cannot be captured. Therefore, the effect of different atomic structures of crack tips on the fracture of pre-cracked graphene sheets and the related fracture mechanisms at atomic scale (i.e. the stretching, rotation and breaking of covalent bonds) are studied by using fully atomistic simulation (i.e. MD simulation) in this subsection.

\subsubsection{Stress-strain relation of different graphene sheets}

The stress-strain curves of the pre-cracked graphene sheets are presented in Fig. 5. The corresponding atomic configurations at different strains are shown in Figs. 6-8, from which the micro-structure evolution and atomic tensile stress distribution in the crack propagation process can be known. From Fig. 5, it can be found that the effective Young's modulus is 0.9TPa for the armchair sheet with u-type crack tip, $0.88 \mathrm{TPa}$ for the armchair sheet with $\omega$-type crack tip and $0.84 \mathrm{TPa}$ for the zigzag sheets. So the armchair sheet can be softened a little more due to the effect of $\omega$-type crack tip structure while the effect of crack tip structures on the effective elastic properties of zigzag sheets are almost the same. For the armchair sheets with u-type and $\omega$-type crack tips, their stress reach the maximum $65.73 \mathrm{GPa}$ and $71.19 \mathrm{GPa}$ at critical strains of $8.77 \%$ and $9.98 \%$, respectively. While for the zigzag sheets with u-type and $\omega$-type crack tips, their stress reach the maximum $57.4 \mathrm{GPa}$ and $70.95 \mathrm{GPa}$ at critical strains of $7.65 \%$ and $10.26 \%$, respectively. Therefore, for the graphene sheets with the same chirality, the fracture stress and strain of the 
sheets with $\omega$-type crack tip are larger than those of the ones with u-type crack tip. For the graphene sheets with u-type crack tip, the fracture strength of the armchair sheet is larger than that of the zigzag sheet, but this difference is narrowed between the armchair and zigzag sheets with $\omega$-type crack tip.
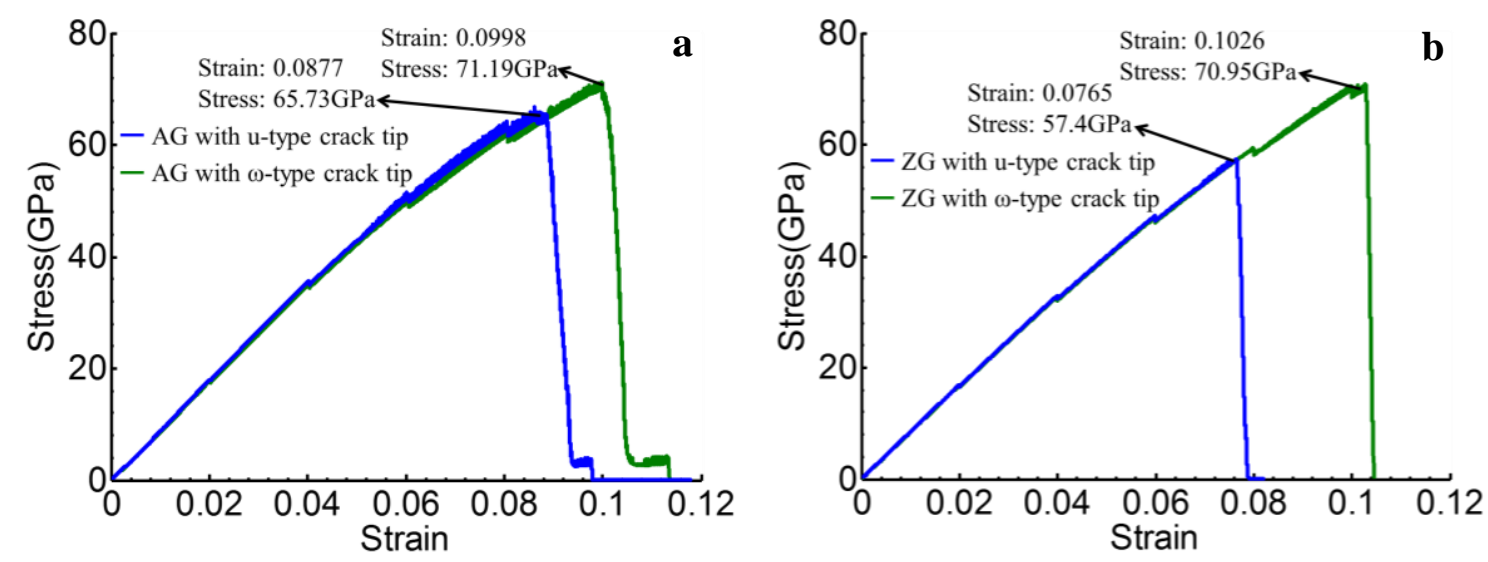

Fig. 5 Stress-strain curves of different pre-cracked graphene sheets. (a) Pre-cracked armchair sheets with u-type and $\omega$-type crack tips, (b) Pre-cracked zigzag sheets with u-type and $\omega$-type crack tips.

In Fig. 5a, it shows that there are three stages during stretching of the armchair sheets, the increasing stress stage, the stress drop-off stage and the stress flow stage. In Fig. 5b, only two stages, the increasing stress stage and the stress drop-off stage, exist during stretching of the zigzag sheets. For each graphene sheet, the stress increases with increasing strain in the increasing stress stage. After stress reaches to its maximum at the critical strain, the stress falls quickly in the stress drop-off stage. Then the stress fluctuates with strain in the stress flow stage for the armchair sheets.

From Figs. 6a-9a which show atomic tensile stress distribution in graphene sheets at critical strain, it can be seen that the atomic stress concentrates at the crack tip and becomes more and more severe with increasing strain for each graphene sheet in its corresponding stress increasing stage. In this stage, the graphene sheets deform almost elastically. Although the length of bonds and angle between bonds are changed, there is no $\mathrm{C}-\mathrm{C}$ bond broken. When the applied strain reaches the critical value, $\mathrm{C}-\mathrm{C}$ bond breaking occurs at the crack tip. Then more and more $\mathrm{C}-\mathrm{C}$ bonds break successively, which leads to continuous expansion of the crack and rapid descending of the stress in the stress drop-off stage.

Figs. $6 \mathrm{~b}, 7 \mathrm{~d}, 8 \mathrm{~b}$ and $9 \mathrm{~b}$ show the atomic stress distribution in graphene sheets near the end of the stress drop-off stage when the crack propagation almost stops. For the armchair sheets, carbon 
chain forms at the end of crack propagation due to the armchair edge effect (See Fig.6b), which also indicates the stress flow stage. Besides, vacancies (carbon polygon rings) can form in the armchair sheet with $\omega$-type crack tip in the crack propagation process (See Figs. $7 \mathrm{~b}$ and d) for the reason that during crack propagation atom micro-vibration in the atom group between the two black lines (See Fig. 7a) can induce the maximum atomic stress to skip around the growing crack tip and cause cleavage of horizontal $\mathrm{C}-\mathrm{C}$ bonds. Then vacancy expands (See Fig. 7c) until it is broken. It is also found that the formation of vacancies is correlated with the size of the region (between the black lines in Figs. 6a and 7a) in which the horizontal $\mathrm{C}-\mathrm{C}$ bonds are likely to break at crack tip when crack initiates. The region size is dependent on the crack tip structure or crack width. Compared with the armchair sheets, there is no obvious carbon chain or carbon polygon ring forming in the crack propagation process of zigzag sheets in Figs. $8 \mathrm{~b}$ and $9 \mathrm{~b}$ mainly due to the difference between armchair and zigzag structures. After breakage of carbon chains, the graphene sheets are fractured completely and the total stress drops to 0GPa. In addition, it can be seen that graphene sheets are mainly fractured in a zigzag way locally and along the original crack direction globally. Such crack propagation pattern should be the consequence of the competition between local and global level fractures.
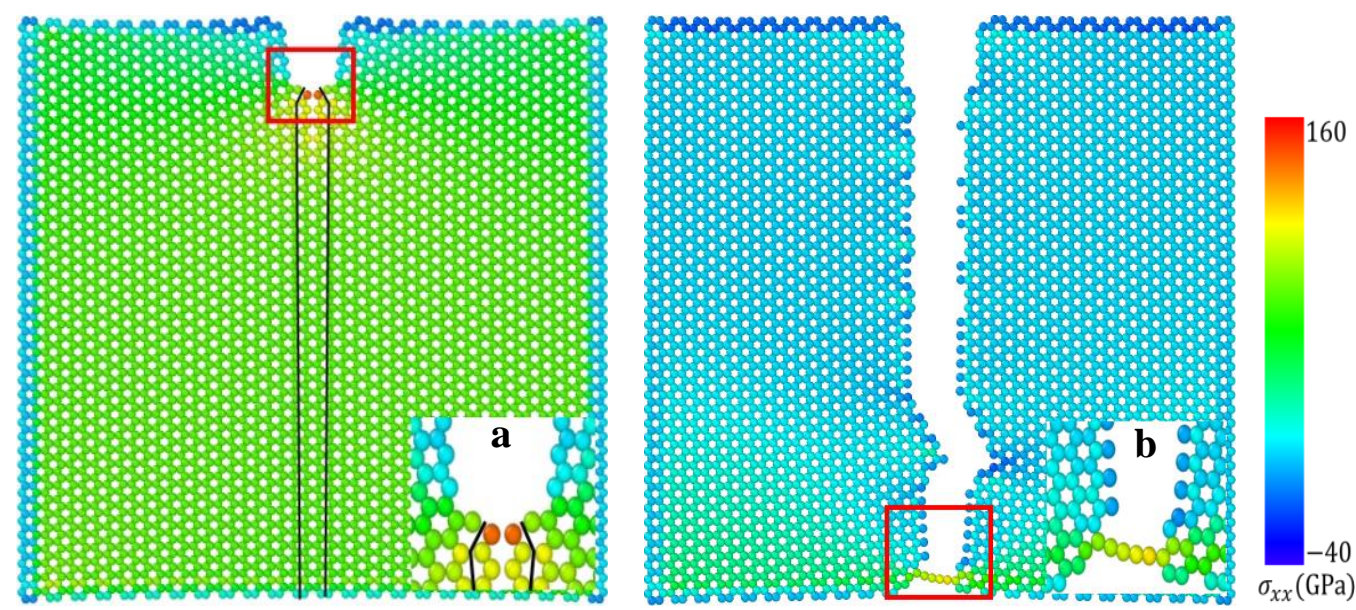

Fig.6 Atomic tensile stress distribution in armchair sheet with u-type crack tip at different strains. (a) Strain=0.0877, (b) Strain=0.0936. The insets show the local atomic configuration in the red rectangles. 

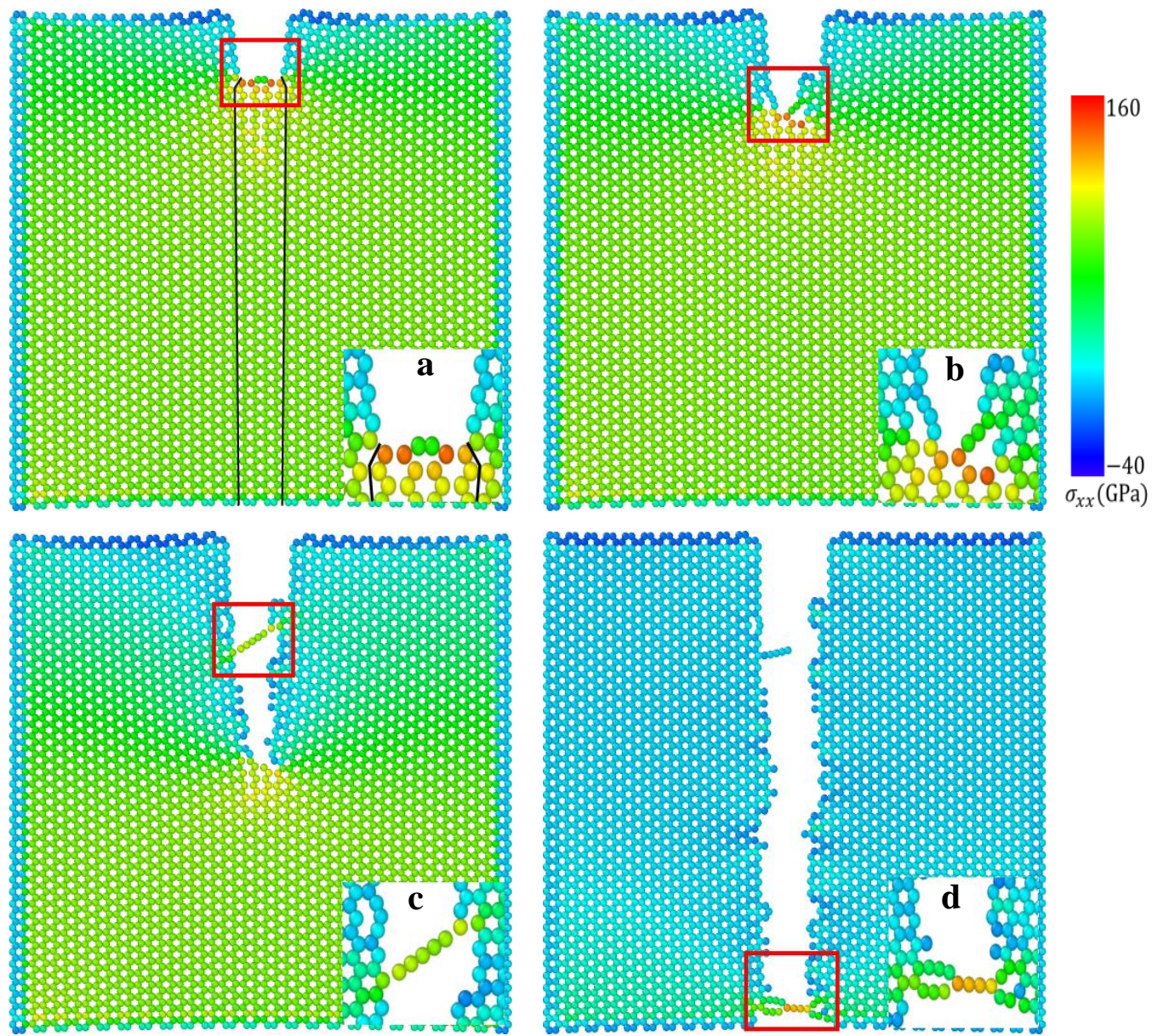

Fig. 7 Atomic tensile stress distribution in armchair sheet with $\omega$-type crack tip at different strains. (a) Strain=0.0998, (b) Strain=0.1009, (c) Strain=0.1023, (d) Strain=0.1053. The insets show the local atomic configuration in the red rectangles.
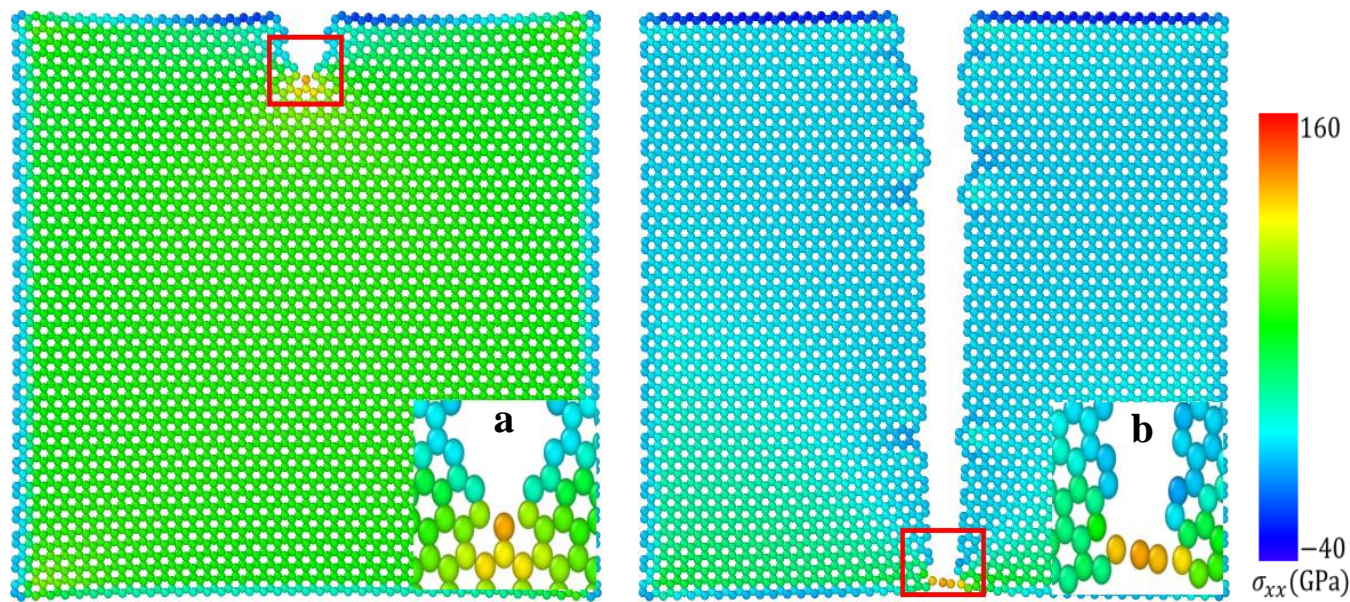

Fig. 8 Atomic tensile stress distribution in zigzag sheet with u-type crack tip at different strains. (a) Strain $=0.0765$, (b) Strain=0.0785. The insets show the local atomic configuration in the red rectangles. 

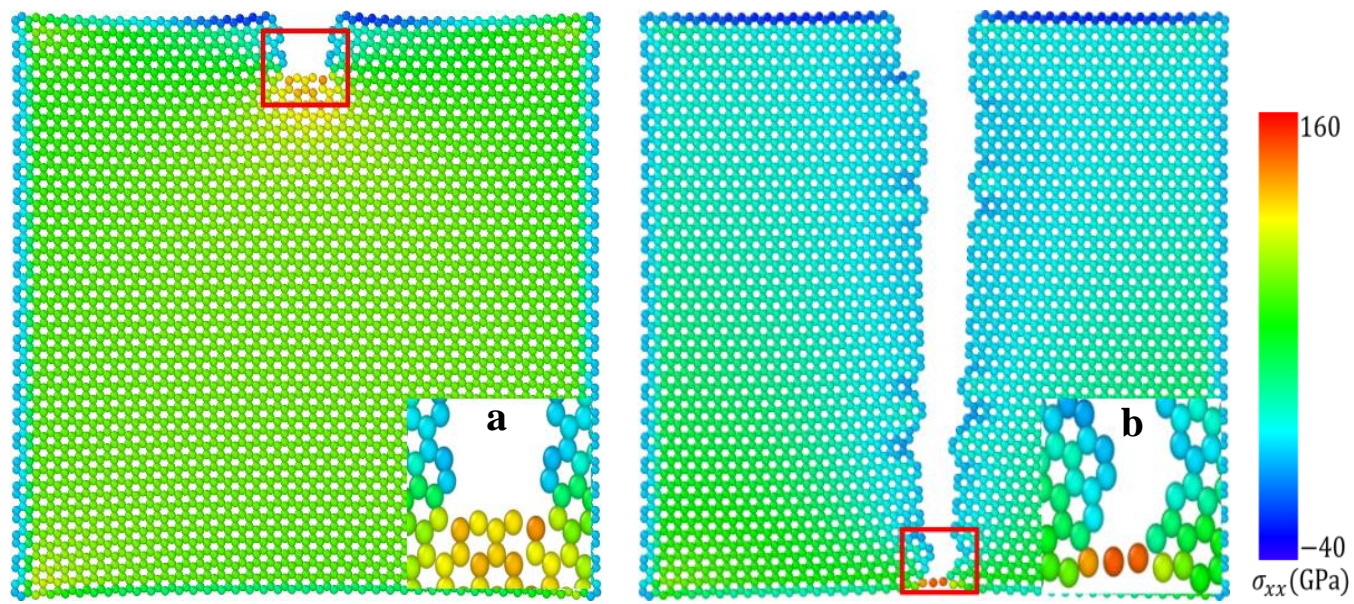

Fig. 9 Atomic tensile stress distribution in zigzag sheet with $\omega$-type crack tip at different strains. (a) Strain=0.1026, (b) Strain=0.1041. The insets show the local atomic configuration in the red rectangles.

\subsubsection{Effect of crack tip structures}

As mentioned earlier, it can be concluded that graphene sheets with different chirality show different mechanical properties and even graphene sheets with the same chirality also show different mechanical properties due to the effect of different crack tip micro-structures. As in armchair or zigzag sheets the crack structures above the crack tips are the same and connect with the crack tip structures through the two atoms in the black circles in Fig. 3 and mainly constrained by the crack tip structures during stretch. Atomic configurations at the crack tip structures between the two atoms in the black circles (see Fig.3) at the initial deformation state and the critical strain are shown in Fig. 10 to clearly know how the structure of crack tip affects the mechanical properties of graphene sheets. Fig. 11 shows the change of length and rotation angle of each bond in every crack tip structure in Fig. 10 along with the increase of global strain before fracture.
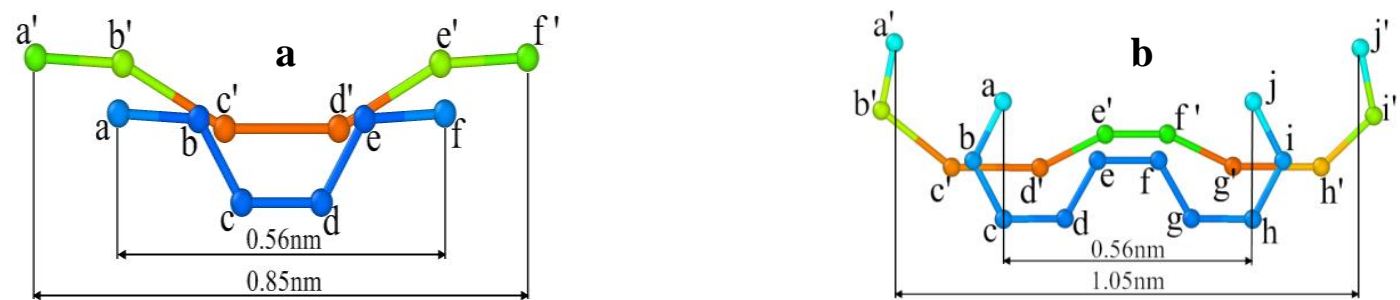

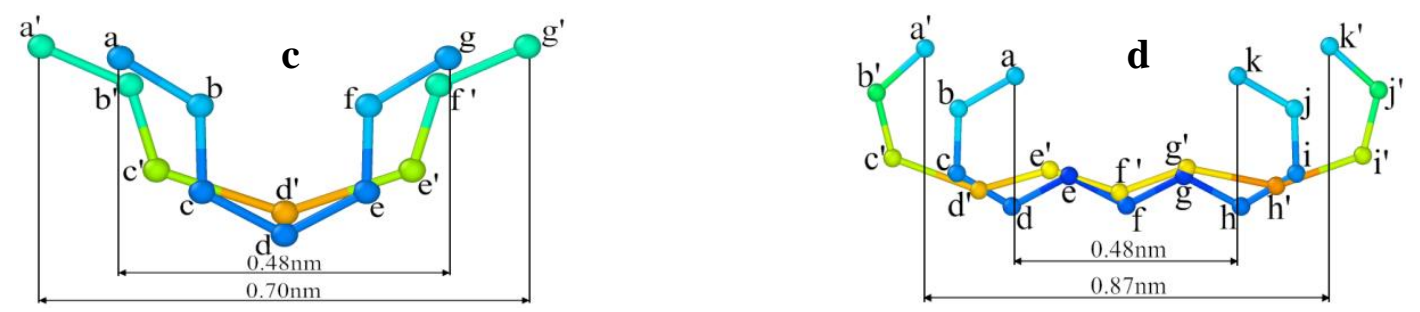

Fig. 10 Atomic configuration at crack tip of each graphene sheet at different strains. (a), (b) u-type and $\omega$-type crack tips in armchair sheets, (c), (d) u-type and $\omega$-type crack tips in zigzag sheets. The crack tip structures at the initial state and the critical strain are characterized by "abc..." and "a'b'c'...", respectively, and colored according to the atomic tensile stress. The two end atoms in each structure are the ones in the black circles in Fig.3.

From Figs. 10 and 11, it can be found that elongation, rotation and movement may occur more or less on the $\mathrm{C}$-C bonds at crack tips during stretch. For this reason, the u-type and $\omega$-type crack tip structures ("ab...ef" and "ab...ij" in Figs. 10a and b) in armchair sheets are stretched by $46.43 \%$ and $87.5 \%$, respectively and the ones ("ab...fg" and "ab...jk" in Figs. 10c and d) in zigzag sheets are stretched by $45.83 \%$ and $81.25 \%$, respectively. At the initial deformation state, the structures are under compression due to the effect of free edge. With stretch continuing, their status changes from compression to tension. Before the critical strain, it can be seen from Fig. 11 that the effect of atomic vibration on the stability of the crack tip structures becomes more and more severe with increasing strain. Figs. 10 and 11 also show that the bonds not parallel to the stretch direction can be rotated due to action of moment arising from the tension force at the two end atoms of each $\mathrm{C}-\mathrm{C}$ bond and the oblique $\mathrm{C}-\mathrm{C}$ bonds can be rotated more in armchair sheets than in zigzag sheets due to their structure difference. For the armchair sheets, Figs.11a and b show that the horizontal C-C bonds (bonds "cd" in Fig. 10a, "cd" and "gh" in Fig. 10b) can be stretched near the cutoff more easily because they are parallel to the stretch direction. For example, the bond "cd" in Fig. 10a is stretched near the cutoff when the global strain reaches only about $5 \%$ while the oblique $\mathrm{C}-\mathrm{C}$ bonds connecting with it are stretched near the cutoff around the critical strain. After this, some oblique $\mathrm{C}-\mathrm{C}$ bonds can be rotated very fast and their rotation angle can reach about $30^{\circ}$ at most. Combining Figs. 10a and b, it also can be found that rotation of the oblique $\mathrm{C}-\mathrm{C}$ bonds (bonds "bc", "de" in Fig. 10a, "bc", "de", "fg" and "hi" in Fig. 10b) can induce the horizontal C-C bonds (bonds "cd" in Fig. 10a, "cd" and "gh" in Fig. 10b) to be moved and due to the movement of such horizontal C-C bonds, the oblique $\mathrm{C}-\mathrm{C}$ bonds can be further rotated as well. For the zigzag sheets, in Fig. 11c the oblique C-C bonds in u-type crack tip structure (bonds "cd" and "de" in Fig. 10c) are stretched near the cutoff around the critical strain. Fig. 11d shows that 
due to the effect of $\omega$-type crack tip structure and the tensile force being transferred from both sides to the inside of the crack tip structure the bonds "cd" and "hi" (See Fig. 10d) can be stretched near the cutoff earlier than the bonds "de" and "ij" (See Fig. 10d) which can be elongated around the cutoff near the critical strain. And Figs. 11c and d show that the oblique C-C bonds can be rotated about $15^{\circ}$ at most. It can be known as well that their rotation can induce small movement of $\mathrm{C}-\mathrm{C}$ bonds. When the global strain reaches the critical value, atomic stress is the maximum at atoms c' and d' in Fig. 10a, and atoms c', d', g' and h' in Fig. 10b. Therefore, bond "c'd" in the u-type crack tip and one of the bonds "c'd"' and "g'h"' in the $\omega$-type crack tip should break first in armchair sheets. Similarly, atomic stress is the maximum at atom d' in Fig. 10c, and atoms d' and h' in Fig. 10d. As a result, one of the bonds "c'd"" and "d'e" in the u-type crack tip and one of the bonds "c'd", "d'e", "g'h"” and "h'i" in the $\omega$-type crack tip should break first in zigzag sheets. Furthermore, it also can be seen in Fig. 10 that the atomic stress at crack tip structures is greater in armchair sheets than in zigzag sheets because of their structure difference that there exist horizontal $\mathrm{C}-\mathrm{C}$ bonds parallel to the stretch direction in armchair sheets but such horizontal C-C bonds do not exist in zigzag sheets. Hence, the crack tip structures can be stretched more in armchair sheets than in zigzag sheets, which is mainly ascribed to the effect of the horizontal C-C bonds in armchair sheets. It also can be concluded that the elongation, rotation and movement (caused by the rotation) of $\mathrm{C}-\mathrm{C}$ bonds make great contribution to the deformation of crack tip structures. In addition, it is evident that the crack tip structures share most part of the strain load applied to the graphene sheets but they also restrict the graphene sheets to bearing larger load due to the defective structures. Besides, there are fewer C-C bonds that can be elongated or rotated in u-type crack tip structures than in $\omega$-type crack tip structures, which causes the $\omega$-type crack tip structures to bear larger strain load than the u-type crack tip structures do. As a result, the graphene sheets with $\omega$-type crack tips show better mechanical properties.
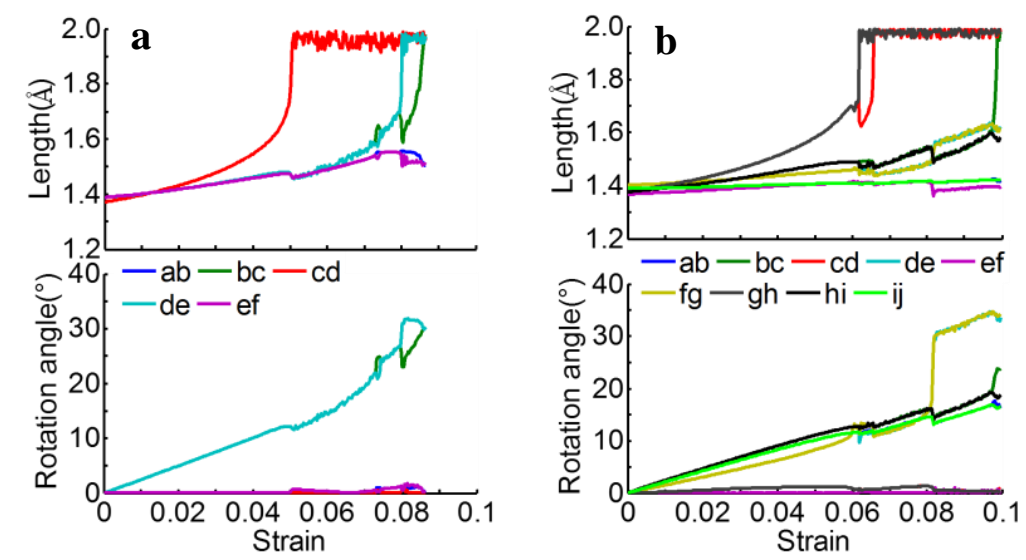

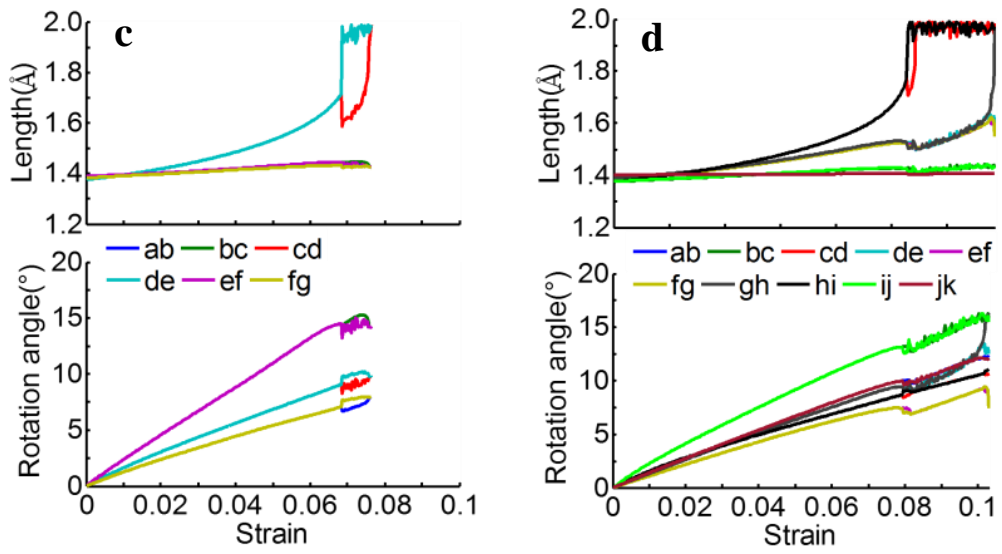

Fig. 11 Change of length and rotation angle of each $\mathrm{C}-\mathrm{C}$ bond in crack tip structures in Fig. 10. (a), (b) u-type and $\omega$-type crack tips in armchair sheets, (c), (d) u-type and $\omega$-type crack tips in zigzag sheets.

\subsubsection{Local stress in the crack propagation process}

To obtain stress state in the crack propagation in the graphene sheets in detail, seven local regions $(\mathrm{R} 1, \ldots, \mathrm{R} 7)$ with size of about $1.3 \mathrm{~nm} \times 1.3 \mathrm{~nm}$ are set along the original crack direction (See Fig. 12). In the course of tension, the tensile stress in each local region is calculated. Fig. 13 shows the relation between the local tensile stress (LS) and the length variation $(\delta)$ of each local region in the stretch direction. In fact, the local tensile stress (LS) and length variation $(\delta)$ can be seen as the traction LS and separation $\delta$ between the two parts at both sides of the local region in horizontal direction.

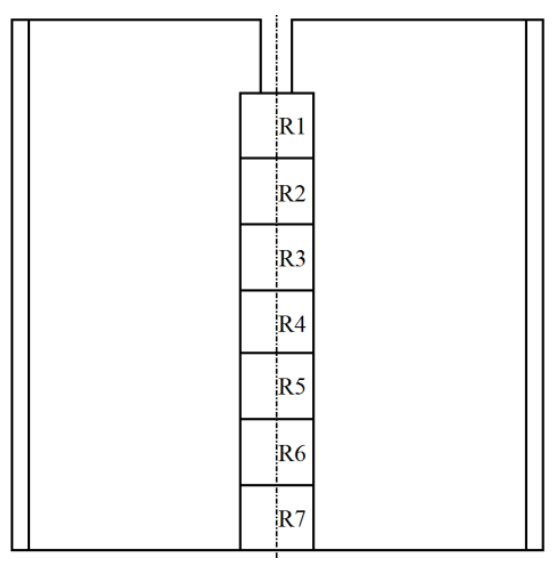

Fig. 12 Local regions along the crack direction in graphene sheet.

From Fig. 13, it can be seen that the maximum local tensile stress is larger than the maximum global tensile stress for all graphene sheets and the maximum local tensile stress is higher in graphene sheets with $\omega$-type crack tip than in graphene sheets with u-type crack tip. Before the 
local tensile stress reaches the maximum, the local regions are stretched almost linearly. After the maximum local stress, the crack tip starts to propagate and the local stress decreases non-linearly with increasing length of local region. For each graphene sheet, the local tensile stress in region $\mathrm{R} 1$ is different from that in other regions for the reason that the initial micro-structure in region $\mathrm{R} 1$ including the crack tip is not identical with that in other regions without any defect. As a result, the region R1 can be stretched more due to the effect of crack tip structure when the local tensile stress reaches the maximum. While the stretch of other regions (from R2 to R7) are relatively small and their difference are very small as well. Therefore, it seems that the local tensile stress reaches the maximum "belatedly" in region R1 and almost simultaneously in other regions in Fig. 13. In fact, they reach the maximum sequentially with the crack propagating from region R1 to R7.
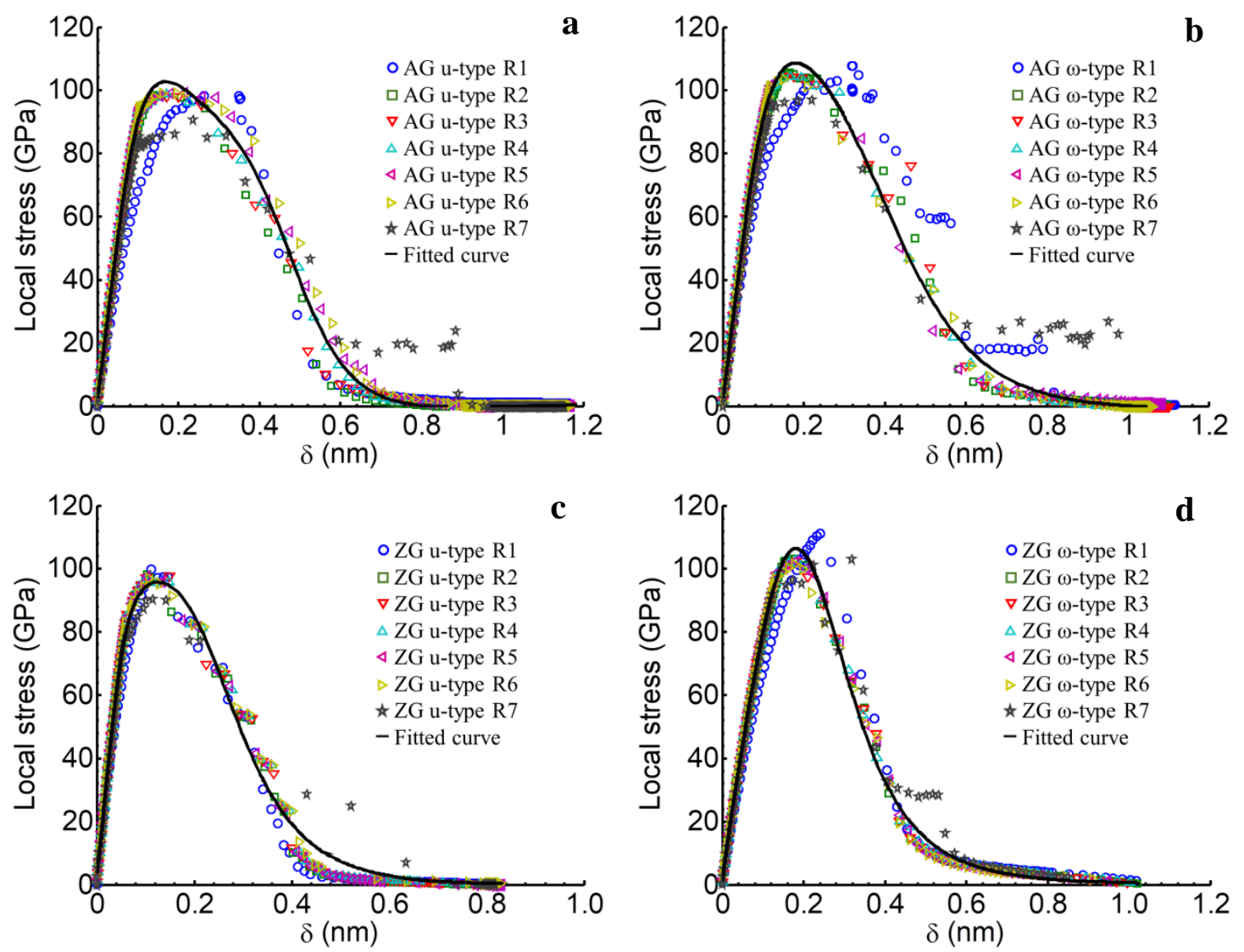

Fig. 13 Local stress in the local regions during stretching. (a), (b) armchair sheets with u-type and $\omega$-type crack tips, (c), (d) zigzag sheets with u-type and $\omega$-type crack tips.

Through comparing the LS- $\delta$ curve in region R1 with the ones in other regions, it can be seen that crack initiation in region $\mathrm{R} 1$ may need more traction or separation than crack propagation does in regions $\mathrm{R} 2$ to $\mathrm{R} 7$. Once the crack propagation starts, steady crack growth process occurs. 
Then at the end of steady crack growth process, in region R7 the traction is not zero after the separation up to $0.6 \mathrm{~nm}$ due to the formation of carbon chain (See Fig.6b). For the armchair sheet with $\omega$-type crack tip, in regions $\mathrm{R} 1$ and $\mathrm{R} 7$ the traction remains after the separation up to $0.6 \mathrm{~nm}$ as well for the reason that carbon chain or polygon ring forms in region $\mathrm{R} 1$ due to the expansion of vacancy (See Fig. 7c) and in region R7 due to the armchair edge effect. When the carbon chain breaks completely, the traction drops to about 0GPa in armchair sheets. And similar with the armchair sheet with u-type crack tip, the traction remains after the separation up to $0.4 \mathrm{~nm}$ in region R7 due to the formation of short carbon chain (See Figs. 8b and 9b) in zigzag sheets. Because the carbon chain is not as obvious as that in armchair sheets, the traction drops to 0GPa very soon in zigzag sheets. Therefore from descriptions above, it can be concluded that the crack propagation process can be characterized by the relation between the traction LS and the separation $\delta$ in the damage zone.

\section{Conclusions}

PD and MD simulations have been performed to study the deformation and fracture mechanisms of pre-cracked single-layer graphene sheets under mode I loading condition. Although the PD simulation results such as the fracture form show good consistency with the MD ones, the related mechanisms at atomic scale cannot be revealed by using the PD theory. From the MD simulation results, some conclusions can be drawn as follow: different crack tip micro-structures have big influence on the mechanical properties of the graphene sheets. The fracture strength and strain of the pre-cracked graphene sheets with $\omega$-type crack tip are larger than the ones with u-type crack tip due to the effect of crack tip structures since there are more C-C bonds in $\omega$-type crack tip structures that can be elongated and rotated. For the sheets with u-type crack tip, the maximum stress of the armchair one is higher than the zigzag one and such difference is narrowed between the sheets with $\omega$-type crack tip. In the crack propagation process, carbon chain and carbon polygon ring can form in armchair sheets due to the armchair edge effect and the difference between armchair and zigzag structures. The formation of polygon ring is related to the crack tip structure and the crack width. Furthermore, the crack propagation process in the damage zone can be characterized by the relation between the local tensile stress and the local stretching, and the current results also present the mechanisms in the crack propagation process in detail. This work also shows that the combination of PD and MD methods can provide a significant insight of multiscale modeling.

\section{Acknowledgment}


This work was fully supported by Science and Technology Innovation Commission of Shenzhen Municipality (JCYJ20160229165310679), Natural Science Foundation of Zhejiang province (LQ13A020002, LY13A020003) and National Natural Science Foundation of China (11372264, 11372281). The authors are grateful for the financial support.

\section{Data availability statement}

The raw/processed data required to reproduce these findings cannot be shared at this time as the data also forms part of an ongoing study.

\section{References}

[1] Grigorieva I V, Novoselov K S, Geim A K, et al. Electric Field Effect in Atomically Thin Carbon Films[J]. Science, 2004, 306(5696): 666-669.

[2] Geim A K, Novoselov K S. The rise of graphene[J]. Nature Materials, 2007, 6(3): 183-191.

[3] Novoselov K S. Nobel lecture: Graphene: Materials in the flatland[J]. Reviews of Modern Physics, 2011, 83(3): 837.

[4] Lee C, Wei X, Kysar J W, et al. Measurement of the elastic properties and intrinsic strength of monolayer graphene[J]. Science, 2008, 321(5887): 385-388.

[5] Ovid'ko I A. Review on grain boundaries in graphene. Curved poly-and nano-crystalline graphene structures as new carbon allotropes[J]. Rev. Adv. Mater. Sci, 2012, 30: 201-224.

[6] Ovid'ko I A. Mechanical properties of graphene[J]. Rev. Adv. Mater. Sci., 2013, 34: 1-11.

[7] Ranjbartoreh A R, Wang B, Shen X, et al. Advanced mechanical properties of graphene paper[J]. Journal of Applied Physics, 2011, 109(1): 014306.

[8] Ponomarenko L A, Schedin F, Katsnelson M I, et al. Chaotic Dirac billiard in graphene quantum dots[J]. Science, 2008, 320(5874): 356-358.

[9] Bolotin K I, Sikes K J, Jiang Z, et al. Ultrahigh electron mobility in suspended graphene[J]. Solid State Communications, 2008, 146(9): 351-355.

[10] Neto A H C, Guinea F, Peres N M R, et al. The electronic properties of graphene[J]. Reviews of Modern Physics, 2009, 81(1): 109.

[11] Molitor F, Güttinger J, Stampfer C, et al. Electronic properties of graphene nanostructures[J]. Journal of Physics: Condensed Matter, 2011, 23(24): 243201.

[12] Balandin A A, Ghosh S, Bao W, et al. Superior thermal conductivity of single-layer graphene[J]. Nano letters, 2008, 8(3): 902-907.

[13]Balandin A A. Thermal properties of graphene and nanostructured carbon materials[J]. Nature Materials, 2011, 10(8): 569-581.

[14]Biró L P, Lambin P. Grain boundaries in graphene grown by chemical vapor deposition[J]. New Journal of Physics, 2013, 15(3): 035024.

[15] Stoller M D, Park S, Zhu Y, et al. Graphene-based ultracapacitors[J]. Nano letters, 2008, 8(10): 3498-3502.

[16]El-Kady M F, Strong V, Dubin S, et al. Laser scribing of high-performance and flexible graphene-based electrochemical capacitors[J]. Science, 2012, 335 (6074): 1326-1330.

[17]Zhang J, Tian T, Chen Y, et al. Synthesis of graphene from dry ice in flames and its application in supercapacitors[J]. Chemical Physics Letters, 2014, 591: 78-81.

[18] Wu J, Becerril H A, Bao Z, et al. Organic solar cells with solution-processed graphene transparent electrodes[J]. Applied Physics Letters, 2008, 92(26): 263302.

[19] Kim K S, Zhao Y, Jang H, et al. Large-scale pattern growth of graphene films for stretchable 
transparent electrodes[J]. Nature, 2009, 457(7230): 706-710.

[20] Bae S, Kim H, Lee Y, et al. Roll-to-roll production of 30-inch graphene films for transparent electrodes[J]. Nature Nanotechnology, 2010, 5(8): 574-578.

[21]Lee Y, Bae S, Jang H, et al. Wafer-scale synthesis and transfer of graphene films[J]. Nano Letters, 2010, 10(2): 490-493.

[22] Lee S K, Kim B J, Jang H, et al. Stretchable graphene transistors with printed dielectrics and gate electrodes[J]. Nano letters, 2011, 11(11): 4642-4646.

[23] Withers F, Bointon T H, Craciun M F, et al. All-graphene photodetectors[J]. ACS Nano, 2013, 7(6): 5052-5057.

[24] Wassei J K, Kaner R B. Graphene, a promising transparent conductor[J]. Materials Today, 2010, 13(3): 52-59.

[25]Li X, Wang X, Zhang L, et al. Chemically derived, ultrasmooth graphene nanoribbon semiconductors[J]. Science, 2008, 319(5867): 1229-1232.

[26] Al-Aqtash N, Li H, Wang L, et al. Electromechanical switching in graphene nanoribbons[J]. Carbon, 2013, 51: 102-109.

[27] Schedin F, Geim A K, Morozov S V, et al. Detection of individual gas molecules adsorbed on graphene[J]. Nature materials, 2007, 6(9): 652-655.

[28]Lee H L, Yang Y C, Chang W J. Mass detection using a graphene-based nanomechanical resonator[J]. Japanese Journal of Applied Physics, 2013, 52(2R): 025101.

[29] Kim R H, Bae M H, Kim D G, et al. Stretchable, transparent graphene interconnects for arrays of microscale inorganic light emitting diodes on rubber substrates[J]. Nano letters, 2011, 11(9): 3881-3886.

[30] Rokni H, Lu W. A continuum model for the static pull-in behavior of graphene nanoribbon electrostatic actuators with interlayer shear and surface energy effects[J]. Journal of Applied Physics, 2013, 113(15): 153512.

[31]Rafiee M A, Rafiee J, Wang Z, et al. Enhanced mechanical properties of nanocomposites at low graphene content[J]. ACS nano, 2009, 3(12): 3884-3890.

[32] Rafiee M A, Lu W, Thomas A V, et al. Graphene nanoribbon composites[J]. ACS nano, 2010, 4(12): 7415-7420.

[33] Young R J, Kinloch I A, Gong L, et al. The mechanics of graphene nanocomposites: a review[J]. Composites Science and Technology, 2012, 72(12): 1459-1476.

[34] Yang Y, Rigdon W, Huang X, et al. Enhancing graphene reinforcing potential in composites by hydrogen passivation induced dispersion[J]. Scientific reports, 2013, 3.

[35] Kim Y, Lee J, Yeom M S, et al. Strengthening effect of single-atomic-layer graphene in metal-graphene nanolayered composites[J]. Nature communications, 2013, 4.

[36] Kam K, Scarpa F, Adhikari S, et al. Graphene nanofilm as pressure and force sensor: A mechanical analysis[J]. physica status solidi (b), 2013, 250(10): 2085-2089.

[37]Reddy C D, Ramasubramaniam A, Shenoy V B, et al. Edge elastic properties of defect-free single-layer graphene sheets[J]. Applied Physics Letters, 2009, 94(10): 101904.

[38] Kiselev S P, Zhirov E V. Molecular dynamics simulation of deformation and fracture of graphene under uniaxial tension[J]. Physical Mesomechanics, 2013, 16(2): 125-132.

[39] Wang M C, Yan C, Ma L, et al. Effect of defects on fracture strength of graphene sheets[J]. Computational Materials Science, 2012, 54: 236-239.

[40] Sun X, Fu Z, Xia M, et al. Effects of vacancy defect on the tensile behavior of graphene[J]. Theoretical and Applied Mechanics Letters, 2014, 4(5): 051002.

[41]Zhu J, He M, Qiu F. Effect of Vacancy Defects on the Young's Modulus and Fracture Strength of Graphene: A Molecular Dynamics Study[J]. Chinese Journal of Chemistry, 2012, 30(7): 1399-1404.

[42]Zhao H, Aluru N R. Temperature and strain-rate dependent fracture strength of graphene[J]. Journal of Applied Physics, 2010, 108(6): 064321.

[43]Zhang Y Y, Gu Y T. Mechanical properties of graphene: Effects of layer number, temperature 
and isotope[J]. Computational Materials Science, 2013, 71: 197-200.

[44] Tsai J L, Tzeng S H, Tzou Y J. Characterizing the fracture parameters of a graphene sheet using atomistic simulation and continuum mechanics[J]. International Journal of Solids and Structures, 2010, 47(3): 503-509.

[45]Zhang Z, Wang X, Lee J D. An atomistic methodology of energy release rate for graphene at nanoscale[J]. Journal of Applied Physics, 2014, 115(11): 114314.

[46] Dewapriya M A N, Rajapakse R, Phani A S. Molecular Dynamics Simulation of Fracture of Graphene[C]//ICF13. 2013.

[47]Le M Q, Batra R C. Single-edge crack growth in graphene sheets under tension[J]. Computational Materials Science, 2013, 69: 381-388.

[48]Le M Q, Batra R C. Crack propagation in pre-strained single layer graphene sheets[J]. Computational Materials Science, 2014, 84: 238-243.

[49] Wang C, Han Q, Xin D. Fracture analysis of single-layer graphene sheets with edge crack under tension[J]. Molecular Simulation, 2015, 41(4): 325-332.

[50]Zhang P, Ma L, Fan F, et al. Fracture toughness of graphene[J]. Nature communications, 2014, 5.

[51]Fang T H, Chang W J, Yang J C. Molecular dynamics study on mechanical properties of graphene sheets with a hole[J]. Digest Journal of Nanomaterials and Biostructures, 2014, 9(3): 1207-1214.

[52]Fang T H, Chang W J, Lin K P, et al. Molecular dynamics analysis for fracture behavior of graphene sheets with V-shaped notches under tension[J]. NANO, 2014, 9(08): 1450087.

[53] Xu M, Tabarraei A, Paci J T, et al. A coupled quantum/continuum mechanics study of graphene fracture[J]. International journal of fracture, 2012, 173(2): 163-173.

[54]Lohrasebi A, Amini M, Neek-Amal M. The effects of temperature and vacancies on dynamics of crack in graphene sheet[J]. AIP Advances, 2014, 4(5): 057113.

[55]Parashar A, Mertiny P. Effect of van der Waals interaction on the mode I fracture characteristics of graphene sheet[J]. Solid State Communications, 2013, 173: 56-60.

[56] Silling S A. Reformulation of elasticity theory for discontinuities and long-range forces[J]. Journal of the Mechanics and Physics of Solids, 2000, 48(1): 175-209.

[57] Oterkus E, Diyaroglu C, Zhu N, et al. Utilization of Peridynamic Theory for Modeling at the Nano-Scale[M]//Nanopackaging: From Nanomaterials to the Atomic Scale. Springer, Cham, 2015: 1-16.

[58] Martowicz A, Staszewski W J, Ruzzene M, et al. Peridynamics as an analysis tool for wave propagation in graphene nanoribbons[C]//Sensors and Smart Structures Technologies for Civil, Mechanical, and Aerospace Systems 2015. International Society for Optics and Photonics, 2015, 9435: 94350I.

[59]Madenci E, Oterkus E. Peridynamic theory and its applications[M]. New York: Springer, 2014.

[60] Madenci E. Peridynamic integrals for strain invariants of homogeneous deformation[J]. ZAMM - Journal of Applied Mathematics and Mechanics/Zeitschrift für Angewandte Mathematik und Mechanik, 2017, 97(10): 1236-1251.

[61]Liu X, He X, Wang J, Sun L, Oterkus E. An ordinary state-based peridynamic model for the fracture of zigzag graphene sheets[J]. Proc. R. Soc. A, 2018, 474: 20180019.

[62]Zhao H, Min K, Aluru N R. Size and chirality dependent elastic properties of graphene nanoribbons under uniaxial tension[J]. Nano letters, 2009, 9(8): 3012-3015.

[63]Plimpton S. Fast parallel algorithms for short-range molecular dynamics[J]. Journal of computational physics, 1995, 117(1): 1-19.

[64]Brenner D W, Shenderova O A, Harrison J A, et al. A second-generation reactive empirical bond order (REBO) potential energy expression for hydrocarbons[J]. Journal of Physics: 
Condensed Matter, 2002, 14(4): 783.

[65]Nosé S. A unified formulation of the constant temperature molecular dynamics methods[J]. The Journal of Chemical Physics, 1984, 81(1): 511-519.

[66] Swope W C, Andersen H C, Berens P H, et al. A computer simulation method for the calculation of equilibrium constants for the formation of physical clusters of molecules: Application to small water clusters[J]. The Journal of Chemical Physics, 1982, 76(1): 637-649.

[67] Stukowski A. Visualization and analysis of atomistic simulation data with OVITO-the Open Visualization Tool[J]. Modelling and Simulation in Materials Science and Engineering, 2010, 18(1): 015012.

[68] Omeltchenko A, Yu J, Kalia R K, et al. Crack front propagation and fracture in a graphite sheet: a molecular-dynamics study on parallel computers[J]. Physical review letters, 1997, 78(11): 2148. 